Vietnam Journal of Mechanics, VAST, Vol. 28, No. 1 (2006), pp. 1 -9

\title{
DANG DINH ANG: NONLINEAR ANALYSIS AND MECHANICS (ON THE OCCASION OF HIS EIGHTIETH BIRTHDAY)
}

\author{
NGuYen Van DaO ${ }^{1}$, NGUyen Dung ${ }^{2}$ \\ ${ }^{1}$ Vietnam National University, Hanoi \\ 2 Institute of Applied Mechanics, VAST
}

Professor Dang Dinh Ang is having his eightieth birthday this year. In honor of the occasion, the Institute of Applied Mechanics and the Institute of Mechanics of The Vietnamese Academy of Science and Technology, and the Laboratoire de Méchanique des Solides, Ecole Polytechnique Paris, with the participation of Vietnam Society of Mathematics, Vietnam Association of Mechanics will jointly organize an International Conference on "Nonlinear Analysis and Engineering Mechanics Today" at the Institute of Applied Mechanics, Vietnamese Academy of Science and Technology, December 11-14, 2006 in Hochiminh City, Vietnam.

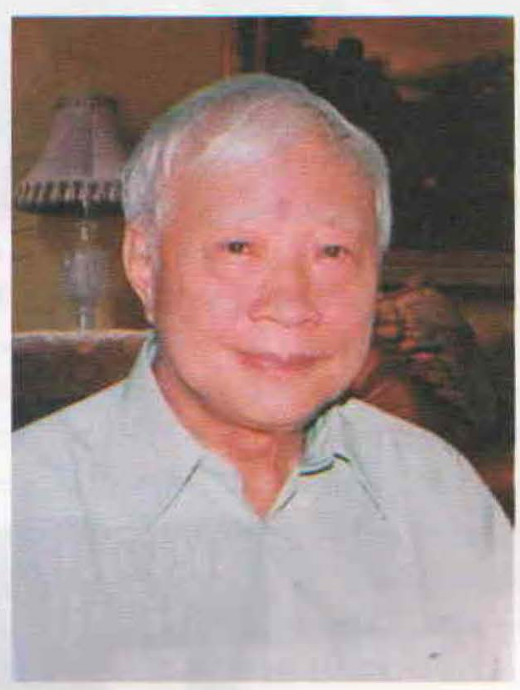

On the occasion of his seventieth birthday, the article Dang Dinh Ang: The nust seventy years "Acta Mathematica Vietnamica" 20 No. 3 (1995) by N. H. Anh, N. X. Sanh and T. D. Van gave delightful details about the scientific career and family life of this eminent scientist. We present below some more details.

Professor Ang was born on 16 March 1926 in Thuy Duong, Chuong My, Ha Tay. After his secondary education in Hanoi, he went to the University of Kansas (KU) to study Aeronautical Engineering. After graduating from $\mathrm{KU}$ in 1955, he went to Caltech (California Institute of Technology) for graduate studies and obtained his Ph.D in Aeronautics and Mathematics in 1958. He became a doctoral fellow in Aeronautics, Caltech 1958-1960, after which he was appointed Head of The Mathematics Department of Saigon University, which position he held until 1975 at which date he became Director of The Analysis Laboratory of Hochiminh City University of Science. He was appointed Professor of Mathematics in 1980.

Dang Dinh Ang has held visiting appointments in some famous international institutions in the United States and in Europe: 
Senior Research Fellow in Aeronautics, then in Geophysics (Caltech 1962, 1964), Associate Geophysicist (UCLA, 1964), Visiting Professor of Mathematics, University of Utah (1970,1988), Université D’Orléans (1987), Instituto per le Applicazioni del Calcolo Mauro Picone in Rome (1984), Université Paris-Nord (1993), DFG Visiting Researcher, Free University Berlin (1989), Visiting Researcher (Math) University of Tokyo. Has lectured at Ecole Polytechnique Paris, University of Cambridge, Banach Center of Mathematics, was member of the organizing Committee for a Workshop on Applications of Mathematics at the Banach Center (1986).

Has directed 12 doctoral theses in Nonlinear Analysis - Mechanics, one of these was presented at Ecole Polytechnique Paris, another was presented at Universit Paris-Nord.

Professor Ang's career is a harmonious synthesis of Nonlinear Analysis and Mechanics. As early as in 1955, he won a prize of the Institute of Aeronautical Sciences in Fort Worth, Texas for his paper on compressible viscous flow. More recently, in 1997, one of the pioneers of Fracture Mechanics K.B.Broberg, in his paper "Early theories of dynamic fracture" in the Anniversary Volume in honor of George R.Irwin's 90th birthday, referred to a problem on dynamic fracture first considered by Fillipov in the Soviet union, then by Maue (1954) in West Germany, and, to quote him "A further illustration of the interest shown in the 1950's for dynamic problems involving stationary cracks is given by the occurrence of a doctor's thesis in the California Institute of Technology by D.D.Ang, who considered the same problem as Maue and used the Wiener-Hopt technique after solution of dual integral equations (Ang 1958). Thus, within a decade, essentially the same problem was solved in the Soviet Union, West Germany and United States, most independently of each other" .

On this occasion, we wish him good health and strength for the continuation of his work.

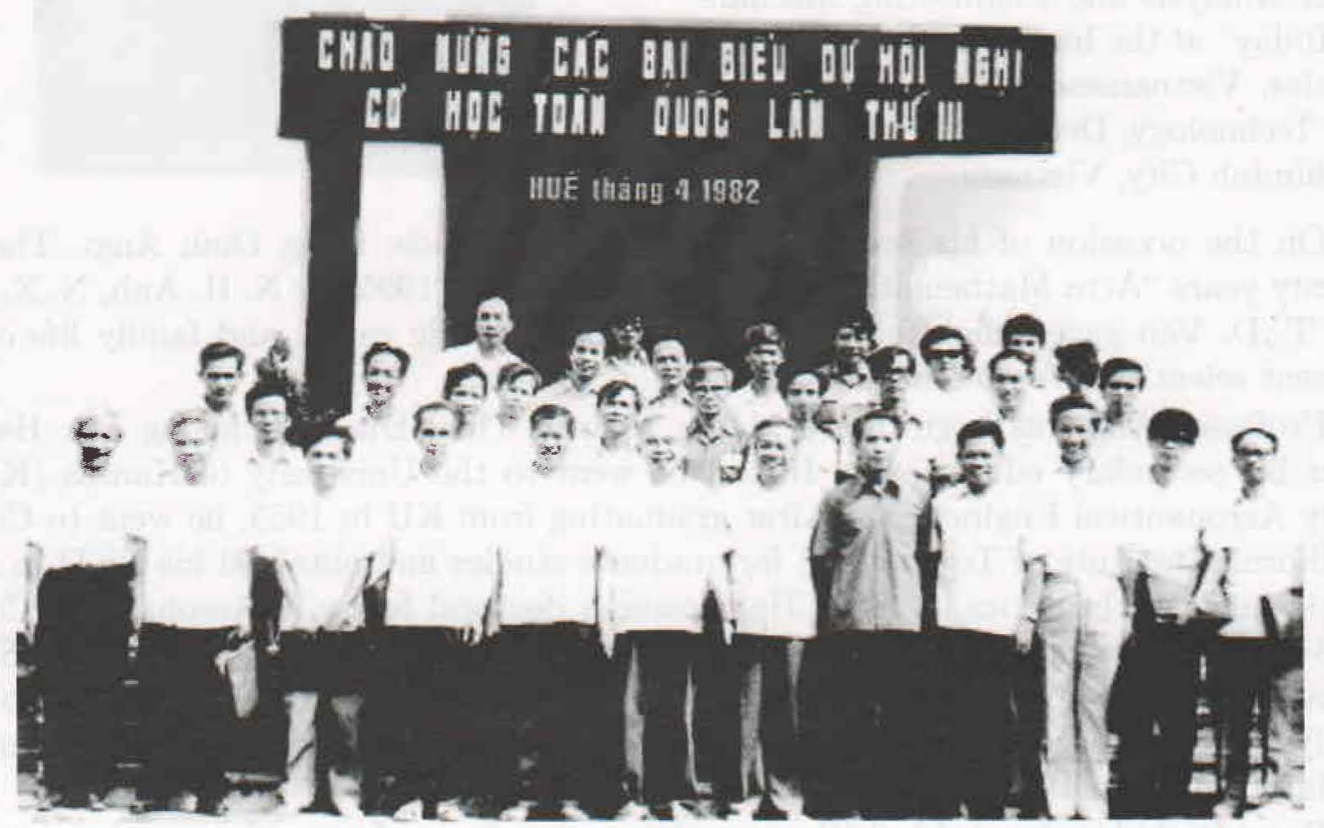

The $3^{\text {rd }}$ National Conference on Mechanics 1982 (in Hue) 


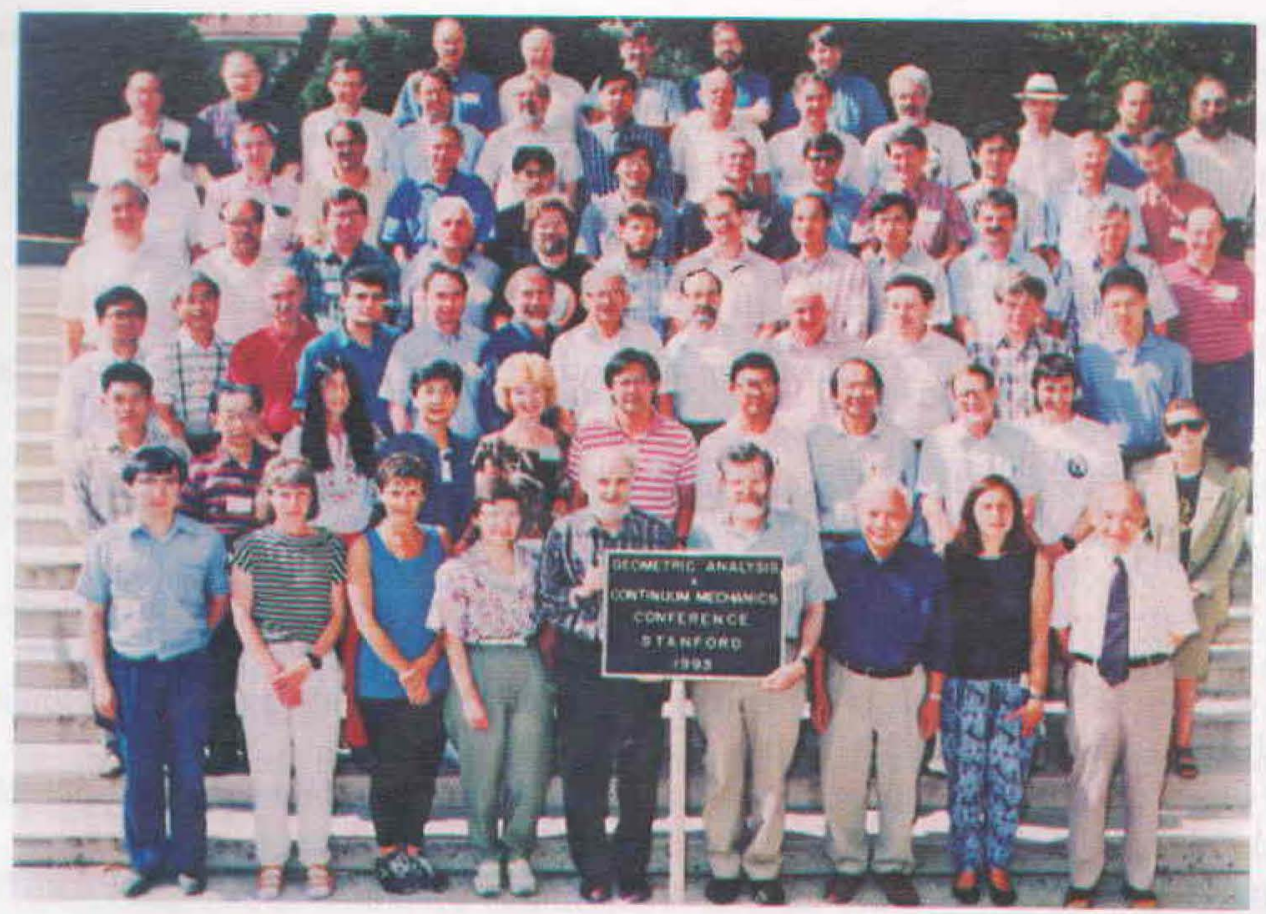

International Conference on Geometric Analysis \&6 Mechanics 1993 (Stanford, USA)

Back row: H. Mittlemann, M. O'Neill, A. Acker, M. Grüter, K. Brauchmann. 6th row: K. Ranger, D. Smith, F. Sauvigny, B. Turkington, R. Gulliver, H. Chang, R. Lauffer, H.-C.Im Hof, U. Hornung, C. Gerhardt, N. Korevaar, K. Lancaster. 5th row: F. Almgren, M. Emmer, W. Borchers, L. Slobozhanin, H. Kozono, T. Miyakawa, G. Galdi, K. Pileckas, J. Heywood, T. Ogawa, J. T. Beale, S. Luckhaus. 4th row: E. Zeidler, A. Myshkis, F. Brulois, A. Koshelev, M. Beeson, J. McCuan, T. Vogel, W. Xie, V. Liu, E. Miersemann, G. Knightly, D. Siegel. 3th row: J. F. Hwang, K. Kenmotsu. F. Milinazzo, B. Fischer, J. Spruck, A. Elcrat, H. Beckert, S. Portnoy, V. Solonnikov, V. Oliker. H. Wente, T. Wan. 2th row: J. T. Chen, T. Otsuki, M. F. Bidaut-Veron, H. Morimoto, B. Kuper, P. Li, L.F. Tam, W. H. Huang, J. Serrin, J. Taylor, P. Pucci. Front row: A. N. Wang, M. Schonbek, M. Padula, E. Portnoy, R. Finn, P. Concus, D. D. Ang, U. Müller, I. D. Chang

\section{SELECTED SCIENTIFIC PUBLICATIONS DANG DINH ANG}

We have presented the essential features of Professor Ang's contributions in Nonlinear Analysis-Mechanics. For reference's sake, we reproduce below a list of his selected scientific publications

1. Displacement thickness in compressible viscous flow, Memoire presented to the Institute of Aeronautical Sciences IAS Fort Worth Texas (USA) 1955.

2. (with M. L. Williams) Some radiation problems in elastodynamics, GALCIT (1957)

3. (with M. L. Williams) On the stress Distribution at the Base of a stationary Crack. J. Appl. Mech. 24 (1957) 109-114. 
4. (with M.L.Williams) The dynamic stress field due to an extensional dislocation. Proc. Midwestern Conf. Solid Mech. (USA) 1959.

5. Radiation waves from a line load moving along a crack, J. Math. Phys. 38 (1960).

6. Transient motion of a line load on the surface of an elastic half-space, Quart. App. Math 18. (1960).

7. (with M. L. Williams) Diffraction of scalar elastic waves by a semi-infinite strip, GALCIT (1961).

8. (with M. L. Williams) Diffraction of vector elastic waves by a semi-infinite strip, GALCIT (1961).

9. (with M. L. Williams) A finite crack in an orthotropic medium, J. App. Mech (1961).

10. (with M. L. Williams) Diffraction of scalar elastic waves by a semi-infinite crack, GALCIT (1962).

11. (with M. L. Williams) Diffraction of vector elastic waves by a semi-infinite crack, GALCIT (1963).

12. (with Es. Folias and M. L. Williams) A finite crack in a plate on an elastis foundation, J Appl. Mech. 03- APM-1 (1963).

13. (with L. Knopoff) Diffraction scalar elastic waves by a finite strip, Proc. US Nat1 Academy of Sci. 51 No. 3 (1964).

14. (with L. Knopoff) Diffraction of vector elastic waves by a finite strip, Proc. US Natl Academy of Sci. 51 No. 3 (1964).

15. (with L. Knopoff) Diffraction of scalar elastic waves by a finite crack, Proc. US Natl Academy of Sci. 51 No. 3 (1964).

16. (with L. Knopoff) Diffraction of vector elastic waves by a finite crack, Proc. US Natl Academy of Sci. 51 No. 3 (1964).

17. (with D. E. Daykin) A note on successive Approx. Mathematika 14 (1967).

18. (with Mal and Knopoff) Diffraction of elastic waves by a penny-shaped strip, Proc. Cambridge Phil. Soc. 64 (1968).

19. A nonlinear temperature problem as a finite strip, Proc. Amer. Math. Soc. $20 \# 2$ (1969)

20. (with D. E. Daykin and T. K. Sheng) On Schoenberg's rational polygon problem J. Australian Math. Soc Vol. IX (1969) pp 337-344.

21. A convolution equation on a half-line J. Math. Analysis and Appl. 24 No. 3 (1968). Correction Ibid 29 no 2 (1970).

22. A convolution equation on a compact interval J. London. Math. Soc. (2) No. 2 (1970)

23. Note on a theorem of Wiener and Pitt, Mathematika 17 (1970).

24. (with L. Knopoff) A two-Phase Stefan problem with melting point gradient. Publ. \# 801, Institute of Geophysics, UCLA, 1971.

25. (with L. Knopoff) A note on $\mathrm{L}_{1}$ - approximation by exponential polynomials and Laguerre polynomials J. Approximation Theory 6 (1972).

26. (with Vu Trong Tuan) An elementary proof of the Morse-Palais Lemma. Proc. Amer. Math. Soc 39 No 3 (1973).

27. (with Le Hoan Hoa) On contractive operators in Hilbert spaces Math. Proc. Cambridges Phil. Soc. 85 (1979). 
28. (with Vu Trong Tuan) A representation theorem for differentiable functions. Proc. Amer. Soc. 75 (1979).

29. Degree of set-valued vector fields and its applications J. Math. Analysis and Appl. 80 (1981).

30. (with Dinh Ngoc Thanh) A probabilistic analogue of the Bohnenblust-Karlin fixed point theorem. Revue Roumaine Math. Pures et Appl. 26 No. 4 (1981).

31. (with D. M. Duc, D. N. Thanh) Relative topological degree of set-valued compact vector fields and its applications. J.Math. Anal. Appl. 80 (1981), No. 2, 406-432.

32. (with Le Hoan Hoa) On a fixed point theorem of Krasnoselskii and triangle contractive operators Fund. Math. CXX (1984) 77-98.

33. Stabilized approximate solutions of certain integral equations of first kind in contact problems of Elasticity Intern. J. Fracture (1984).

34. The inverse time problem for a diffusion equation Proc. Workshop Math. Probl. Geophysics, IGU. Venice. Dec. 1984.

35. Stabilized approximate solutions of the inverse time problem for a parabolic evolution equation, J. Math. Analysis and Appl. Vol. 111, No. 1, 1985.

36. (with D. D. Hai) Regularization of Abel's integral equation Proc. Roy. Soc. Edinburgh (1987)

37. (with Alain Pham, N. D.) Mixed problem for a semi-linear wave equation with nonhomogeneous boundary conditions Nonlinear Analysis TMA. Sept. 1988.

38. (with Alain Pham, N. D.) Strong solutions of a quasilinear wave equations with nonlinear damping SIAM J. Math. Analysis. March 1988.

39. (with Alain Pham, N. D.) On a strongly damped wave equation SIAM J. Math. Anal. Nov 1988.

40. (with Keinert and Stenger) A two-phase nonlinear Stefan problem with melting point gradient: a constructive approach J. Appl. and Comp. Math. (1988).

41. (with P.N.Dinh) On some viscoelastic strongly damped nonlinear wave equations. Nonlinear hyperbolic equations theory, computation methods, and applications (Aachen, 1988), 499-509, Notes Numer. Fluid Mech., 25, Vieweg, Braunschweig, 1989.

42. (with Stenger and Lund) Complex variables and regularization methods for the inverse Laplace transform Math. Comput. Oct 1989, 589-608.

43. (with Folias, Keinert and Stenger) Indentation and viscoplastic flow: a free boundary problem Intern. J. Fracture (a special issue in honor of ML Williams) 1989.

44. (with Alain Pham, N. D.) Some Viscoelastic wave Equations Intern. J. Fracture, 1989.

45. A pseudo-parabolic equation Proc. Iternl Conf. Diff. Eqns, Oberwolfach, 1989.

46. (with D.D.Hai) On nonsmooth solutions of Abel's integral equation. Differential Integral Equations 3 (1990), No. 2, 397-400.

47. (with K.Schmitt and L.K.Vy) Noncoercive variational inequalities: some applications, Nonlinear Analysis, TMS 15 No. 6 (1990), 497-512.

48. (with T. Thanh) A nonlinear pseudo-parabolic equation, Proc. Roy. Soc. Edinburgh 114 A (1990). 119-133.

49. On backward parabolic equation: A critical survey of some current methods, Numerical Analysis and Math. Modeling, Publication Banach Math. International Center 24, Warsaw 1990. pp. 509-515. 
50. (with K. Schmitt and L. K. Vy) Variational inequalities and the contact of plates, Lect. Notes Pure and Appl. Math. M. Dekker 133 (1991), pp 1-19.

51. (with R. Gorenflo) A nonlinear Abel integral equation. Lect. Notes Control and Information Sciences. Editors Hoffmann-Krabs, Springer (1991), 26-37.

52. (with E. S. Folias and F. Keinert) Penetration mechanics: predicting the location of a viscoplatic boundary and its effects on the stresses, International J. Solids and Structures 28 (1991).

53. (with L. K. Vy) Stablized approximation of parameters in a variational inequality, 1991.

54. (with D. D. Hai) On the backward parabolic equation, Ann. Polon. Math. LII (1991).

55. (with R.Gorenflo) A nonlinear Abel integral equation Lect. Notes on Control and Information Sciences, Springer-Verlag, Hoffmann-Krabs Edits, 1991, pp 26-37.

56. (with R. Gorenflo and L. K. Vy) Backus-Gilbert regularization for a moment problem. Preprint, Series A, Math, Institute, Berlin Free University, 1991.

57. (with E. S. Folias and M. L. Williams) The bending stress in a cracked plate on an elastic foundation, J. Appl. Mechanics paper No. 63 - APN - 1, 1963.

58. (with D. D. Hai) Regularization of a generalized Abel integral equation, Applicable Analysis 45 pp 424-432, 1992.

59. (with L. K. Vy) Regularized solutions of a three-dimensional inverse scattering problem, Inverse Problems 8 (1992), 499-507.

60. (with L. K. Vy) Identification of coefficients in an inhomogeneous Helmholtz equation by asymptotic regularization, Inverse Problems 8 (1992), 308-523.

61. (with R. Gorenflo and D. D. Hai) Regularization of generalized Abel integral equation, Applicable Analysis 45 (1992), 331-332.

62. (with K. Schmitt and L. K. Vy) P-coercive variational inequalities and unilateral problems for Von Karman's equations, WSSIA A 1, World Scientific Publ. (1992), 15-29.

63. (with K. Schmitt and L. K. Vy) P-coercive variational inequalities and unilateral problems for Von Karman's equations, Lakshmikantham Ann. Volume in 1992.

64. Stress singularities in a cracked orthotropic plate, Internatl J. Fracture, 1992.

65. (with L. K. Vy) Contact of a plate with an elastic body: an interface model, J. Elasticity, 1992

66. (with D. N. Thanh and V. V. Thanh) Regularized solutions of a Cauchy problem for the Laplace equation in an irregular strip, J. Integ. Eqs and Applics (1993), 429-441.

67. (with L. K. Vy and R. Gorenflo) A Regularization method for the moment problem, In "Inverse Problem: Principles and Applications", Math. Research Vol. 74, Akademic Verlag 1993, 37-44.

68. (with L. K. Vy) Stabilized approximation of parameters in a variational inequality, Model Optimization in Exploration Geophysics 6 (1993), 1-16.

69. (with L. K. Vy and R. Gorenflo) Backus-Gilbert regularization of a moment problem, Preprint No. A-7 98, Institute of Mathematics, Freie Universitat Berlin, 1993.

70. (with L. K. Vy) Stabilized approx. of parameters of a parabolic variational inequality Ninth International Seminar on model Optim. and Exploration geophysics Friedr. Vieweg and Sons, 1993. 
71. Model optimization in Exploration Geophysics Vol. 3 (A.Vogel edit.) 1993.

72. (with D. D.Trong) Asymptotic behavior of solutions of the equations of compressible heat conductive flows, Proceedings, International Conf. "Geometric Analysis and Continumm Mechanics" in honor of Robert Finn, Standford U., Calif, August 1993, International Press, 1995.

73. (with D. D. Trong) Coefficient identification for a parabolic equation, Inverse Problems 10 (1994), 733-752.

74. (with D. D. Trong) Global solutions of a free boundary problem in heat and mass transfer for polymeric materials, Results in Mathematics 26 (1994), 155-177.

75. (with R. Gorenflo) Degenerate and nondegenerate nonlinear Abel integral equations of the first kind, Nonlinear Analysis TMA 22, No. 1 (1994), 63-72.

76. (with L. K. Vy) Contact of a plate with an elastic body: an interface model, J. Elasticity 35 (1994), pp 1-26.

77. (with L. K. Vy) More on applications and extensions of P-coercive variational inequalities, Acta Math. Vietnam 19 (1994), 51-70.

78. (with R. Gorenflo) Degenerate and non degenerate nonlinear Abel integral equations of first kind, 1994.

79. (with N. Cam) Remarks on the identification problem for elliptic equations, Proceedings, International Workshop "Inverse Problems" HoChiMinh City, January 1995, editors: Ang-Gorenflo et Al., Public. No. 2, HoChiMinh Math. Soc., 1995, 21-23.

80. (with R. Gorenflo and D. N. Thanh) Regularization for a two dimensional inverse Stefan problem, Proceedings, International Workshop "Inverse Problems" HoChiMinh City, January 1995, editors: Ang-Gorenflo et al. Public, No. 2, HoChiMinh Math. Soc., 1995, pp. 45-54.

81. (with L. K. Vy) Domain identification for harmonic functions, Acta Applicandae Mathematicae 38 (1995), 217-238.

82. (with L. K. Vy) Some extensions and applications of P-coercive variational inequalities, Proceedings, First World Congress of Nonlinear Analysts, De Gruyter, Berlin (1995).

83. (with L. K. Vy) Contact of a plate with an elastic body, Zeitschrift f. Ang. Mathematik u. Mech. (ZAMM) 75 No. 2 (1995), 115-126.

84. (with L. K. Vy) Frictional contact of an elastic body with a rigid support, Nonlinear Analysis TMA 25 No. 4 (1995), 339-343.

85. (with L. K. Vy) Domain Identification for Harmonic Functions. Acta Applicandae. Math. 38, 1995, 217-238

86. (with D. D. Trong) More on coefficient identification for a parabolic equation. Inverse problems and applications to geophysics, industry, medicine and technology (HoChiMinh, 1995), 170-173, Publ.HoChiMinh City Math. Soc., 2, HoChiMinh City Math. Soc., HoChiMinh City, 1995.

87. (with V. V. Thanh) Extrapolation in gravimetry: a moment problem. Numerical analysis and applications (HoChiMinh City, 1995), 11-14, Publ. HoChiMinh City Math.Soc., 4, HoChiMinh City math. Soc., HoChiMinh City, 1995.

88. (with V. V. Thanh) A regularized solution of an inverse problem of gravimetry. HoChiMinh City Math.Soc. 1995.

89. (with B. D. Khanh, C. D. Khanh) A numerical resolution of the exterior inverse Radon transform problem, 53-59, Publ. HoChiMinh City, Math.Soc. (1995). 
90. (with D. D. Trong and Yamamoto): Unique Continuation and Identification of Boundary of an Elastic Body: Journal of Inverse and Ill-posed Problems, No. 6, 1996, 417-428.

91. (with R.Gorenflo and D.N.Thanh) Determination of shape and mass density gravity anomaly measurements: some uniqueness results, preprint A 14-97, F. Mathematik und Informatik, Serie A - Mathematik, Free University Berlin.

92. (with R. Gorenflo and L. K. Vy) Uniqueness theorem for a nonlinear integral equations of gravimetry, Proc.World Congress of Nonlinear Analysts (1992), Walter de Gruyter, Berlin (1996) pp. 2423-2430.

93. Cauchy problem and domain identification for elliptic equations and systems: a restricted survey pp 1-14, proc. $5^{\text {th }}$ Vietnam Math. Conf. Hanoi September 17-20, 1997.

94. (with R. Gorenflo and L. K. Vy) Regularization of a nonlinear integral equation of gravimetry, J.Inv.Ill-posed Problems 5 \# 2 (1997) 101-116.

95. Cauchy's problem and domain identification for Elliptic equations and systems: A restricted Survey, Proceedings, Fifth Vietnamese Math. Conf Hanoi, Sep. 1997. Editors: P. K. Anh, H. H. Khoai, N. V. Mau, N. K. Son, D. T. Thi, N. D. Tien, D. L. Van.

96. (A. Pham, N. D and D. N. Thanh) Regularization of a 2-dimensional two phase inverse Stefan problem, Inverse Problems 13 (1997) 607-619.

97. (with K. Schmitt and L. K. Vy) A multidimensional analogue of the Denjoy-PerronHenstock-Kurzweil integral, Bull. Belg. Math. Soc.4 (1997) pp. 335-371.

98. (with D. D.Trong) Crack detection by the electric method: uniqueness and approximation, Intern. J. Fracture 93, pp 1-4 (1998).

99. (with D. D. Trong) Crack Detection by the Electric Method: Uniqueness and Approximation. Internatinal J. of Fracture 93 (1998), 63-86.

100. (with Ikehata, M., Trong and M. Yamamoto) Unique Contituation for a Stationary Isotropic Lame System with Variable Coefficients. Comm. In Partial Diff. Eqns. 23 (1998), 371-385.

101. (with N. H. Nghia and N. C. Tam) Regularized solutions of a Cauchy problem for the Laplace equation in an irregular layer: a 3-D model. Acta. Math. Vietnam 23, 1998, 65-74.

102. (with D. D. Trong) Domain identification for nonlinear elliptic equation. Zeitschrift fur Analysis und ihre Anwendungen 17 (1998) 4, 1021-1024.

103. (with N. V. Nhan, D. N. Thanh) A nonlinear integral equation of gravimetry: uniqueness and approximation by linear moments. Vietnam J. Math 27 (1999), No. 1, 61-67.

104. (with D.D.Trong and Yamamoto) Identification of Cavities Inside 2-dimensional Heterogeneous Isotropic Elastic Body. J. of Elasticity 56 (1999), 199-212.

105. (with R.Gorenflo and D. D. Trong) A multidimensional Hausdorff moment problem: Regularization by finite moments, ZAA 18 (1999) No.1, pp.13-25.

106. (with D. N. Thanh and V. V. Thanh) Identification of mass inhomog. from surface gravity anomalies, Geophysics. J. Int. (2000) 495-498.

107. Detection of cavities in solids and solutions of elliptic equations and systems. Vietnam J. Math. 29:1 (2001) 1-14. 
108. (with D. N. Thanh and V. V. Thanh) Identification of mass inhomogeneity from surface gravity anomalies, Geophysical J. International (Royal Astronomical Society) Vol.143 No., Nov-2002.

109. (with D.D.Trong) Crack Detection in Plane Semilinear Elliptic Equations in the Plane: the Zero Flux Case. Zeitschrift fur Analysis und ihre Anwendungen 19 (2001), 109-120.

110. (with L. K. Vy) Contact of a viscoelastic body with a rough rigid surface and identification of coefficients, J. Inverse and Ill-Posed Problems 9 No.1 pp.1-20 (2001).

111. (with N. Dung and D. D. Trong) Boundary identification for an elastic solid partly immersed in a liquid, Vietnam J. Mechanics 24 (2002) No.1 pp.1-13.

112. (with R. Menniken, D. N. Thanh and D. D. Trong) Cavity Detection by Electric Method: The 3-dimensional Case (in ZAMM) (2003-2004).

113. (with N. Dung, N. V. Huy and D. D. Trong) Uniqueness of elastic continuation in a semilinear elastic body, Vietnam J. Mechanics 252003 No.1 pp.1-8.

114. (with P. T. Trinh) Inverse problems in geosciences, J. Geology series B, No.1314/1999 pp.194-199.

115. (with D. D. Trong, M. Yamamoto) A Cauchy problem for elliptic equations: quasireversibility and error estimates. Vietnam J. Math (2004).

116. (with D. D. Trong) Identification of cavities in a three dimensional elastic body. Z.Anal. Anwendungen 23 (2004), No.2, 407-422.

117. (with L. K. Vy, D. D. Trong) Reconstruction of an analytic function from a sequence of values: existence and regularization. Finite or infinite dimensional complex analysis and applications, 127-142, Adv. Complex Anal. Appl., 2, Kluwer Acad. Publ. 2004.

118. (with F. Folias, F. Keinert and F. Stenger) Viscoelastic flow due to penetration: a free boundary value problem Structural Integrity: Theory and Experiment, Kluwer Acad. Publishers. 1989.

119. (with C. D. Khanh, M. Yamamoto) A Cauchy like problem in plane elasticity: a moment theoretic approach, Vietnam J. Math 32 SI, pp.19-32, 2004.

120. (with D. D. Trong) Identification of cavities in a 3-D elastic body, Vietnam J. Math 33: 4, pp 407-422, 2004.

121. Stress field in an elastic strip in frictionless contact with a rigid stamp, Vietnam J. Math 33:4, pp. 469-475, 2005.

\section{MONOGRAPHS}

1. Lý thuyết tích phân (Theory of Integration), NXB Giáo Dục, 1997.

2. Nhập môn giài tích (Introduction to Analysis), NXB Giáo Dục, 1997

3. (Với Trịnh Anh Ngọc, Ngô Thành Phong) Nhập môn cơ học (Introduction to Mechanics), NXB Đại học Quốc Gia TP. HCM, 2003.

4. (With R. Gorenflo, Vy K. Le and D. D. Trong) Moment Theory and some Inverse problems in Potential theory and Heat conduction. Springer, Berlin, Heidelberg, 2002

5. (With Vy Khoi Le) Variational Inequalities: Contact Problems in Mathematical Engineering and some Related Free Boundary Like Problems (Submitted to Chapman \& Hall/CRC, 2005) 